\title{
Brane-world creation and black holes
}

\author{
Jaume Garriga \\ IFAE, Departament de Fisica, Universitat Autonoma de Barcelona, 08193 Bellaterra (Barcelona), Spain \\ Misao Sasaki \\ Department of Earth and Space Science, Graduate School of Science, Osaka University, Toyonaka 560-0043, Japan \\ and Department of Physics, School of Science, University of Tokyo, Tokyo 113-0033, Japan
}

(Received 23 December 1999; published 27 July 2000)

\begin{abstract}
An inflating brane world can be created from "nothing" together with its anti-de Sitter (AdS) bulk. The resulting space-time has compact spatial sections bounded by the brane. During inflation, the continuum of KK modes is separated from the massless zero mode by the gap $m=(3 / 2) H$, where $H$ is the Hubble rate. We consider the analogue of the Nariai solution and argue that it describes the pair production of "black cigars" attached to the inflating brane. In the case when the size of the instantons is much larger than the AdS radius, the 5-dimensional action agrees with the 4-dimensional one. Hence, the 5D and 4D gravitational entropies are the same in this limit. We also consider thermal instantons with an AdS black hole in the bulk. These may be interpreted as describing the creation of a hot universe from nothing or the production of AdS black holes in the vicinity of a pre-existing inflating brane world. The Lorentzian evolution of the brane world after creation is briefly discussed. An additional "integration constant" in the Friedmann equation-accompanying a term which dilutes like radiation-describes the tidal force in the fifth direction and arises from the mass of a spherical object inside the bulk. In general, this could be a 5-dimensional black hole or a "parallel" brane world of negative tension concentrical with our brane-world. In the case of thermal solutions, and in the spirit of the AdS/CFT correspondence, one may attribute the additional term to thermal radiation in the boundary theory. Then, for temperatures well below the AdS scale, the entropy of this radiation agrees with the entropy of the black hole in the AdS bulk.
\end{abstract}

PACS number(s): $98.80 . \mathrm{Cq}$

\section{INTRODUCTION}

The idea that we may live on a brane propagating in a bulk spacetime of higher dimension is currently being considered in a variety of contexts, ranging from $M$ theory [1] to phenomenological particle physics [2]. In particular, Randall and Sundrum (RS) have proposed a simple and attractive scenario where our brane world is embedded in a 5-dimensional bulk with a negative cosmological constant $\Lambda_{5}[3,4]$. In this scenario, the extra dimension need not be small. In fact it could be infinite and yet we would perceive gravity as effectively 4 dimensional [4-6]. The reason is that the bulk is strongly curved, so that most of its physical volume is within a short distance $l \sim \Lambda_{5}^{-1 / 2}$ from the brane. The picture of a brane world evolving in a larger spacetime brings with it a new perspective for early universe cosmology. In particular, it seems natural to investigate the question of brane-world creation.

The dynamics of the gravitational field on the RS brane has been recently analyzed in $[5,6]$. For the case of a single brane with positive tension, it has been shown that 4D Einstein gravity is recovered on the wall, with some corrections at short distances and at high matter densities [5,6]. For the case of two parallel branes with opposite tension, there are additional scalar interactions corresponding to the brane separation modulus. These give rise to Brans-Dicke type theories on the branes with rather interesting behavior [6] (the case where the dilaton is stabilized has received some attention in [7]). A discussion of gravitational collapse of matter on the brane was given in [8]. It was argued that the resulting "black hole", would be a 5-dimensional object attached to the brane, with an event horizon in the shape of a cigar. This conjecture was confirmed in the analogous (2 $+1)$-dimensional case [9]. In the $(3+1)$-dimensional case, the conjecture is consistent with the form of the weak field created by spherical matter sources on the wall [6], although the non-perturbative 'black cigar' solution has not yet been found. Finally, a great deal of attention has been devoted to cosmological solutions $[10,11]$. In any discussion of gravity on the brane, the issue of boundary conditions in the bulk is quite important. If we are interested in the gravitational field created by matter sources on an asymptotically flat brane, it seems reasonable to impose that the bulk be asymptotically AdS in the past Cauchy horizon and at spacelike infinity, and that there be no incoming graviton flux from the past Cauchy horizon [6]. In the cosmological context, however, it seems much more appropriate to address the question of boundary conditions in the framework of quantum cosmology.

In this paper, we shall consider the quantum creation of an inflating brane world and its subsequent evolution. In Sec. II we shall present the de Sitter-brane instanton. In Sec. III we shall generalize it to find the analogue of the Nariai solution. As we shall see, this solution can be interpreted as describing pair creation of black cigars attached to the inflating brane. In Sec. IV we shall consider "thermal" instantons which contain AdS black holes in the bulk. These may describe the creation of a hot universe from nothing or the production of AdS black holes in the vicinity of a preexisting inflating brane world. Our conclusions are summarized in Sec. V. 


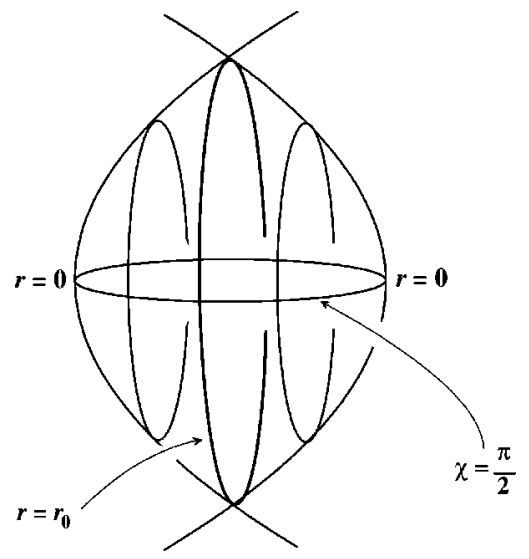

FIG. 1. de Sitter-brane instanton. The thick vertical circle at $r$ $=r_{0}$ represents the 4-sphere brane at which the two identical 5 -dimensional anti-de Sitter spaces are glued. The $\chi=\pi / 2$ hypersurface is the nucleation geometry.

\section{DE SITTER-BRANE INSTANTON}

In 4-dimensional gravity, the simplest description of the birth of the universe involves the de Sitter instanton. This is a 4-sphere which interpolates between a point (or "nothing') at the south pole and a 3-sphere of maximal radius at the equator. The 3-sphere represents the geometry of the universe at the moment of creation. The subsequent cosmological evolution is given by the analytic continuation of the 4-sphere to Lorentzian signature, which gives an inflating spacetime.

This picture can be generalized to the case of a brane world embedded in an AdS bulk. The line element of a 5-dimensional Euclidean AdS space can be written as

$$
d s_{E}^{2}=g_{a b} d x^{a} d x^{b}=d r^{2}+l^{2} \sinh ^{2}(r / l)\left[d \chi^{2}+\sin ^{2} \chi d \Omega_{(3)}^{2}\right] .
$$

Here $d \Omega_{(3)}^{2}$ is the metric on the 3-sphere and $l=$ $\left(-6 / \Lambda_{5}\right)^{1 / 2}$ is the AdS radius. A compact brane-world instanton can be constructed by excising the spacetime region at $r>r_{0}$ and gluing two copies of the remaining spacetime along the 4-sphere at $r=r_{0}$. On that hypersurface a brane of tension $\sigma$ is introduced so that Israel's matching conditions [5]

$$
\left.\partial_{r} g_{\mu \nu}\right|_{r=r_{0}}=\left.\frac{8 \pi G_{5}}{3} \sigma g_{\mu \nu}\right|_{r=r_{0}}
$$

are satisfied. Here $G_{5}$ is the 5-dimensional gravitational constant and

$$
\sigma=\frac{3}{4 \pi G_{5} l} \operatorname{coth}\left(r_{0} / l\right)
$$

The greek indices run over the coordinates of the 4-sphere. The result of this cut and paste procedure is illustrated in Fig. 1. Two copies of a spherical patch of AdS bulk are bounded by a common 4-sphere, which is the world sheet of the brane. We may further identify both copies of the bulk by

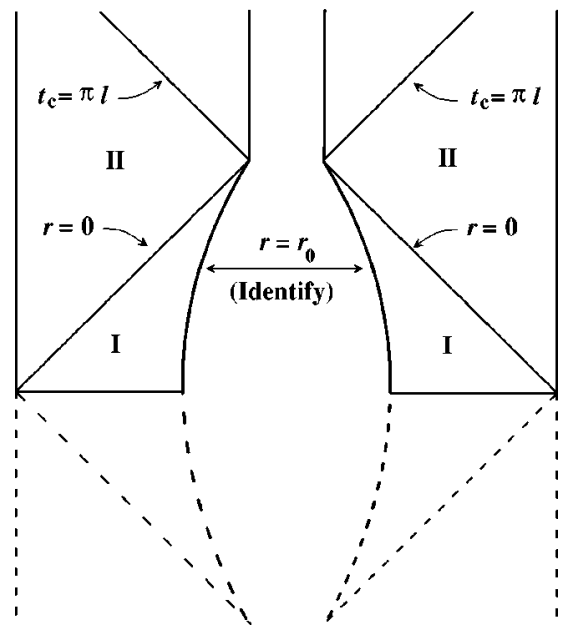

FIG. 2. Conformal diagram of the inflating brane world (at $r$ $=r_{0}$ ) created from nothing. Each point in the figure represents a 3-sphere.

imposing a $Z_{2}$ symmetry, although this identification will not play a prominent role in our discussion.

The instanton in Fig. 1 can be interpreted as a semiclassical path for the creation of a universe from nothing. Cutting the instanton in half, the Euclidean solution interpolates between "nothing," at the south pole, and a spherical brane of radius

$$
H^{-1} \equiv l \sinh \left(r_{0} / l\right)
$$

at the equator. This is completely analogous to the usual 4-dimensional de Sitter instanton, except that in the present case the inside of the brane world is filled with AdS bulk.

The evolution of the brane after creation is given by the analytic continuation of Eq. (2.1) to real time. This continuation is done by means of the substitution $\chi \rightarrow i H t+(\pi / 2)$, which leads to

$$
\begin{aligned}
d s^{2}= & d r^{2}+(l H)^{2} \sinh ^{2}(r / l) \\
& \times\left[-d t^{2}+H^{-2} \cosh ^{2}(H t) d \Omega_{(3)}^{2}\right] \quad\left(r \leqslant r_{0}\right) .
\end{aligned}
$$

The Lorentzian spacetime is represented in Fig. 2.

The brane at $r=r_{0}$ is now an inflating de Sitter space with Hubble rate $H^{-1}$. The line element (2.4) does not cover the whole spacetime, but only the exterior of the Rindler horizon at $r=0$ (region I). This horizon is the light cone emanating from the center of symmetry of the solution. To cover the interior of the light cone (region II), we can make the complexification $r \rightarrow i t_{c}, H t \rightarrow r_{c}-i(\pi / 2)$. In region II, the metric is that of a 5-dimensional open Friedmann-Robertson Walker (FRW) universe:

$$
d s^{2}=-d t_{c}^{2}+l^{2} \sin ^{2}\left(t_{c} / l\right)\left[d r_{c}^{2}+\sinh ^{2} r_{c} d \Omega_{(3)}^{2}\right]
$$

where $r_{c} \sim 1$ corresponds to the scale of spatial curvature. The coordinate $t_{c}$ runs from 0 to $\pi l$. At $t_{c}=\pi l$ the open universe "recollapses." The null surface $t_{c}=\pi l$ is a Cauchy horizon across which we can match our solution to a new 
AdS region or to a new de Sitter-brane region. But in any case, this extended solution is just a mathematical idealization, too simple to represent the real world.

Inflation will only be useful if it comes to an end, giving rise to thermalized regions. For that reason, we should consider the situation where the effective tension $\sigma$ is partly due to the potential energy of an inflaton field $\varphi$ :

$$
\sigma=\sigma_{c}+V(\varphi) .
$$

Here, we have arbitrarily separated from $V$ the constant part,

$$
\sigma_{c}=\frac{3}{4 \pi l G_{5}} .
$$

A brane which has the "critical" tension $\sigma_{c}$ does not inflate in the AdS background [the static Randall-Sundrum flat brane world [4] can be recovered from Eq. (2.4) in the limit $r_{0} \rightarrow \infty$, which from Eq. (2.3) corresponds to $\sigma=\sigma_{c}$ ], so the effective cosmological constant vanishes for $V=0$.

The cosmological evolution of the closed universe after creation can be obtained as follows. As a result of spherical symmetry, the bulk inside a closed brane world will be simply AdS, which is conveniently described in the static chart as

$$
d s^{2}=-A(R) d T^{2}+\frac{d R^{2}}{A(R)}+R^{2} d \Omega_{(3)}^{2},
$$

where $A(R)=\left(1+R^{2} / l^{2}\right)$. The brane-world trajectory can be parametrized as $R=R(t), T=T(t)$. There is much freedom in this parametrization, and we shall choose the gauge in which

$$
\dot{R}^{2}=A(R)^{2} \dot{T}^{2}-A(R) .
$$

With this choice, the metric on the brane is given by

$$
d s_{4}^{2}=-d t^{2}+R^{2}(t) d \Omega_{(3)}^{2} .
$$

The equation of motion for the scale factor $R$ can be obtained from Israel's matching conditions under the assumption of $Z_{2}$ symmetry:

$$
K_{\mu \nu}=-4 \pi G_{5}\left[T_{\mu \nu}-(1 / 3) T g_{\mu \nu}\right],
$$

where $T_{\mu \nu}$ is the energy momentum tensor on the brane and $K_{\mu \nu}$ is the extrinsic curvature. In terms of the normal vector $n_{a}=(\dot{R},-\dot{T}, 0,0,0)$, this is given by $K_{a b}=-\left(g_{a}^{c}-n_{a} n^{c}\right)\left(g_{b}^{d}\right.$ $\left.-n_{b} n^{d}\right) n_{c ; d}$. The angular components are given by

$$
K_{\Omega \Omega^{\prime}}=n_{a} \Gamma_{\Omega \Omega^{\prime}}^{a}=-\dot{T}[A(R) / R] g_{\Omega \Omega^{\prime}} .
$$

Assuming that the energy momentum tensor has the perfect fluid form

$$
T_{\nu}^{\mu}=\operatorname{diag}(-\rho, p, p, p)-\sigma_{c} \delta_{\nu}^{\mu},
$$

where $\sigma_{c}$ is given in Eq. (2.6) and defining

$$
X=\frac{8 \pi G}{3} \rho,
$$

the angular components of Eq. (2.9) give

$$
\left(\frac{\dot{R}}{R}\right)^{2}=\frac{1}{l^{2}}-\frac{A(R)}{R^{2}}+X+\frac{l^{2}}{4} X^{2} .
$$

It can be shown that the temporal components of Eq. (2.9) are in fact redundant. With $A(R)=1+R^{2} / l^{2}$ we have

$$
\left(\frac{\dot{R}}{R}\right)^{2}=-\frac{1}{R^{2}}+\frac{8 \pi G}{3} \rho+l^{2}\left(\frac{4 \pi G}{3} \rho\right)^{2} .
$$

Hence, the standard Friedmann equation for cosmological evolution is recovered [12,22], with a correction term which is negligible at moderately low energy densities $l^{2} X \ll 1$.

Returning our attention to the inflating de Sitter solution, let us now check the existence of the bound state of gravity on the brane and the nature of the spectrum of Kaluza-Klein (KK) excitations. Expanding the metric as $d s^{2}=\left(g_{a b}\right.$ $\left.+h_{a b}\right) d x^{a} d x^{b}$, we shall work in the analogue of the RS gauge,

$$
h_{r r}=h_{r \mu}=h_{\mu}^{\mu}=h_{\mid \nu}^{\mu \nu}=0,
$$

where the vertical stroke indicates a covariant derivative in the 4-dimensional de Sitter space. It is convenient to define $\hat{h}_{\mu \nu}=a^{-1 / 2} h_{\mu \nu}$, where $a(r)=l \sinh (r / l)$, and a conformal radial coordinate $\eta$ through $d r=a d \eta$. In terms of $\eta$ we have

$$
a(\eta)=\frac{l}{\sinh \left(|\eta|+\eta_{0}\right)} \quad(-\infty<\eta<+\infty)
$$

where $\eta_{0}$ is defined by the equation $\sinh \eta_{0}=1 / \sinh \left(r_{0} / l\right)$. The equation of motion for perturbations in the gauge (2.12) can be separated as [13]

$$
\begin{gathered}
-\hat{h}_{\mu \nu}^{\prime \prime}+\frac{\left(a^{\prime \prime 3 / 2}\right)}{a^{3 / 2}} \hat{h}_{\mu \nu}=\frac{m^{2}}{H^{2}} \hat{h}_{\mu \nu}, \\
\left(-\square+2 H^{2}+m^{2}\right) \hat{h}_{\mu \nu}=0 .
\end{gathered}
$$

The Schrodinger equation (2.13) determines the spectrum of masses $m^{2}$, while Eq. (2.14) is the equation of motion for spin two fields of mass $m^{2}$ in a de Sitter space of radius $H^{-1}$. The box indicates the 4-dimensional covariant d'Alembertian.

Equation (2.13) has the obvious normalizable bound state $\hat{h}_{\mu \nu} \propto a^{3 / 2}$, which corresponds to the massless graviton. However, it is clear that the "volcano potential" in Eq. (2.13) approaches the constant $9 / 4$ at $\eta \rightarrow \pm \infty$. Hence, the continuous spectrum of KK excitations starts at

$$
m=(3 / 2) H \text {. }
$$

This is a somewhat special value of the mass in de Sitter space, corresponding to the critically damped case. For smaller mass, long wavelenth perturbations are under- 
damped, whereas for larger mass they have oscillatory behavior. As is well known, de Sitter space behaves in some respects as a system with temperature $T=H / 2 \pi$. The mass gap between the zero mode and the KK modes may be related to the stability of de inflating brane world against thermal excitation of the massive modes. This issue deserves further investigation.

Solutions with two concentrical branes can easily be constructed by inserting a new brane of negative tension,

$$
\tilde{\sigma}=-\frac{3}{4 \pi G_{5} l} \operatorname{coth}\left(r_{1} / l\right)<0,
$$

at some radius $r_{1}<r_{0}$. The region $r>r_{1}$ is excised, and the edges of the two copies of the bulk are again identified along the 4-sphere at $r_{1}$. In the absence of a mechanism stabilizing the interbrane distance, the subsequent cosmological evolution on both branes will be independent (although the evolution of cosmological perturbations will not be so, in general). In particular, the branes will in general drift away or come closer together in the course of evolution.

An important property of our scenario is that the problem of boundary conditions on the past cauchy horizon does not exist. Our brane world is entirely contained in the causal future of the compact hypersurface at which the whole universe appears in the Lorentzian regime. Hence the evolution of the brane world is uniquely determined by the initial data. One can then unambiguously calculate the quantum fluctuations around the classical solution. One can either adopt Hartle and Hawking's no-boundary boundary condition [14] or Vilenkin's tunneling boundary condition [15]. In the 4-dimensional theory, it is known that both boundary conditions give the same fluctuation spectrum [16]. In the present 5-dimensional theory, detailed evaluation of the quantum fluctuation spectrum is left for future study, but the analysis of the KK modes given above indicates that only the massless (graviton) mode contributes. Hence the result would be similar to the case of standard 4-dimensional quantum cosmological inflation, irrespective of the Hartle-Hawking or Vilenkin boundary conditions.

\section{PAIR PRODUCTION OF "BLACK CIGARS"}

As in the case of flat brane worlds, it is possible to find nonlinear generalizations of the zero mode on the brane. With the ansatz

$$
d s^{2}=g_{a b} d x^{a} d x^{b}=d r^{2}+a^{2}(r) \gamma_{\mu \nu} d x^{\mu} d x^{\nu},
$$

where $\gamma_{\mu \nu}$ is the 4-dimensional metric and $a(r)=l \sinh (r / l)$, the 5-dimensional Riemann tensor is given by

$$
\begin{aligned}
R_{r \nu r}^{\mu} & =-l^{-2} \delta_{\nu}^{\mu}, \quad R_{\nu \rho \sigma}^{\mu} \\
& ={ }^{(\gamma)} R_{\nu \rho \sigma}^{\mu}+\cosh ^{2}(r / l)\left(\delta_{\sigma}^{\mu} \gamma_{\nu \rho}-\delta_{\rho}^{\mu} \gamma_{\nu \sigma}\right) .
\end{aligned}
$$

It is then straightforward to show that the 5-dimensional equations of motion, $R_{a b}=-4 l^{-2} g_{a b}$, are satisfied provided that $\gamma_{\mu \nu}$ is a solution of

$$
{ }^{(\gamma)} R_{\mu \nu}=3 \gamma_{\mu \nu} .
$$

Brane solutions can be constructed in the same manner as in the previous section, by excising the region with $r>r_{1}$ and introducing at the boundary a brane of tension given by Eq. (2.3). The metric on the brane will be given by $a^{2}\left(r_{0}\right) \gamma_{\mu \nu}$. The argument is valid both for Euclidean and for Lorentzian metrics. Equation (3.3) can be interpreted as the 4-dimensional Einstein equations on the brane in the presence of a positive cosmological constant:

$$
\Lambda_{4}=\frac{3}{a^{2}\left(r_{0}\right)} \equiv 3 H^{2} .
$$

One solution of Eq. (3.3) is de Sitter space, but any other solution can be chosen. As in the previous section, we may optionally introduce a second brane of negative tension, Eq. (2.15), at some $r_{1}<r_{0}$, excising the region with $r<r_{1}$.

The Euclidean action for the system of two branes of tension $\sigma_{i}$ located at $r_{i}$ and separated by a region of AdS bulk is given by

$$
S_{E}=\frac{1}{16 \pi G_{5}} \int d^{5} x \sqrt{g}\left(2 \Lambda_{5}-\mathcal{R}\right)+\sum_{i} \sigma_{i} a^{4}\left(r_{i}\right) \int d^{4} x \sqrt{\gamma}
$$

where $\mathcal{R}$ is the Ricci scalar. The energy momentum tensor is given by

$$
T_{a b}=-\left(8 \pi G_{5}\right)^{-1} \Lambda_{5} g_{a b}-\sum_{i} \sigma_{i} a^{2} \gamma_{a b} \delta\left(r-r_{i}\right),
$$

where $\gamma_{a b}=0$ when $a$ or $b$ are equal to $r$. Using the trace of the Einstein equations, $\mathcal{R}$ can be eliminated from Eq. (3.4), which gives

$$
S_{E}=-\frac{1}{12 \pi G_{5}} \int d^{5} x \sqrt{g} \Lambda_{5}-\frac{1}{3} \sum_{i} \sigma_{i} a^{4}\left(r_{i}\right) \int d^{4} x \sqrt{\gamma}
$$

Performing the integrals we have

$$
S_{E}=\sum_{i=0}^{1}(-1)^{i}\left(\operatorname{coth}\left(r_{i} / l\right)-\frac{r_{i} / l}{\sinh ^{2}\left(r_{i} / l\right)}\right) S_{E}^{(i)},
$$

where

$$
S_{E}^{(i)}=-\frac{3 V_{4}^{(\gamma)}}{8 \pi G H_{i}^{2}}
$$

Here

$$
G=G_{5} / l
$$

is the 4-dimensional Newton constant (defined in the limit of low energy world or when the effective tension of the brane is close to $\sigma_{c}$ and the matter density is much lower than $\sigma_{c}$ ), $H_{i}=\left[l \sinh \left(r_{i} / l\right)\right]^{-1}$, and $V_{4}^{(\gamma)}$ is the (dimensionless) volume of the manifold with metric $\gamma_{\mu \nu}$. In the case of a single brane of positive tension, only the first term in Eq. (3.6) is present. Also, in the limit where $r_{0} \gg r_{1}, l$, we have $S_{E}$ 
$\approx S_{E}^{(0)}$, which in turn reduces to the usual 4-dimensional action of the instanton with metric $a^{2}\left(r_{0}\right) \gamma_{\mu \nu}$ and cosmological constant $\Lambda_{4}=3 H_{0}^{2}$.

It should be noted that the one-brane 5-dimensional solutions of the form (3.1) will in general be singular. Indeed, from Eq. (3.2), the non-vanishing components of the 5-dimensional Weyl tensor are given by $C^{\mu}{ }_{\nu \rho \sigma}={ }^{(\gamma)} C^{\mu}{ }_{\nu \rho \sigma}$, and therefore

$$
C^{2}={ }^{(\gamma)} C^{2} / a^{4}(r) .
$$

Unless ${ }^{(\gamma)} C^{2}=0$ (as is the case when the brane metric is conformally flat) this invariant diverges in the vicinity of $r$ $=0$. Of course, this singularity will not occur in the case with two branes. A similar divergence occurs in the RandallSundrum solution [4] when we substitute the flat 4-dimensional metric on the brane by any Ricci flat metric $\gamma_{\mu \nu}$ :

$$
d s^{2}=d y^{2}+a^{2}(y) \sigma_{\mu \nu} d x^{\mu} d x^{\nu},
$$

where $a(y)=l e^{|y| / l}$. In this case the divergence is at the AdS horizon, $y \rightarrow \infty$. An exception occurs in the case when $\sigma_{\mu \nu}$ is a 4-dimensional plane wave [17]. The reason is that plane waves are null, and any invariant such as ${ }^{(\sigma)} C^{2}$ vanishes. In the cosmological case, ${ }^{(\gamma)} C^{2}$ does not vanish even for linearized gravity waves. This applies to the zero mode discussed in the previous section, which is therefore singular on the Rindler horizon. However, we do not believe that this indicates any fundamental difficulty. The expansion in modes is just an instrument which is useful in order to find the Green's function of the wave equation. For instance, in the case of flat slices discussed by Randall and Sundrum, the KK modes are singular on the AdS horizon, and yet the gravitational field created by isolated sources on the brane (which is computed by using the Green function of the wave operator) is non-singular $[6,18]$.

Let us now consider the Schwarzschild-de Sitter solution on the brane:

$$
H^{-2} d \sigma^{2}=-F(R) d T^{2}+\frac{d R^{2}}{F(R)}+R^{2} d \Omega_{(2)}^{2} .
$$

Here $F(R)=\left(1-H^{2} R^{2}-2 M / R\right)$ and $M$ is a free parameter. For $H M \ll 1$, the equation $F(R)=0$ has three real solutions. One of them is negative and the other two are positive. The two positive roots correspond to the black hole and cosmological horizons respectively. For $H M \ll 1$ the 5-dimensional solution is analogous to the "black string" described in [8]. At large distances from the brane $\sinh (r / l) \ll(H M)^{-1}$, the physical size of the horizon $a(r) H M$ is much smaller than the AdS radius $l$. In this region, the horizon of the black string locally resembles a cylinder, which is entropically unstable to fragment into an array of spherical horizons which in total have a larger surface area [19].

If the parameter $M$ in Eq. (3.9) is increased, the black hole horizon on the brane increases and the cosmological horizon decreases. The extremal case where both horizons have the same size is the Nariai solution, which has $M=(3 \sqrt{3} H)^{-1}$ and $R=(\sqrt{3} H)^{-1}$. The $R, T$ coordinates become inadequate in this case, since $F$ tends to zero. It is customary to introduce a small parameter $\epsilon$, defined by $27 M^{2} H^{2}=1-3 \epsilon^{2}$, and new coordinates $(\lambda, \psi)$ through $\lambda=\epsilon \sqrt{3} H T, \sqrt{3} H R=(1$ $\left.-\epsilon \cos \psi-\epsilon^{2} / 6\right)$. The black hole horizon is now at $\psi=0$, whereas the cosmological horizon is at $\psi=\pi$ [20]. In the limit $\epsilon \rightarrow 0$, one finds the Nariai solution

$$
d \sigma^{2}=\frac{1}{3}\left[-\sin ^{2} \psi d \lambda^{2}+d \psi^{2}+d \Omega_{(2)}^{2}\right] .
$$

Near this extremal case, the 5-dimensional solution is qualitatively different from the black string. In fact, it appears to be much closer to the black cigar postulated in Ref. [8]. The potentially unstable regime $a(r) H M \ll l$ only arises for $r \ll l$, where the "scale factor" behaves linearly $a \propto r$. In this region, the horizon does not look like a cylinder at all. In fact, the spatial section of the horizon closes off smoothly at $r$ $=0$, and looks like Fig. 1, except that now the vertical circles are 2-spheres rather than 4-spheres. Therefore, the usual argument that the horizon should be entropically unstable, breaking up into small spheres, cannot be applied. Some instability may still be expected near the tip of the cigar, at $r$ $=0$, where $C^{2}$ diverges. We shall argue, however, that the singularity at $r=0$ is rather mild, and may in fact be smoothed out by quantum fluctuations. Note, in this connection, that even for small zero mode perturbations near the de Sitter-brane solution, the invariant $C^{2}$ diverges near $r=0$. Zero point fluctuations corresponding to these modes, however, have finite action and will inevitably occur. Similar fluctuations may be expected to smooth out the tip of the black cigar in the Nariai-brane solution.

As is well known, the 4-dimensional Nariai instanton can be Euclideanized by making the substitution $\lambda \rightarrow i \phi$ in Eq. (3.10). The corresponding Euclidean solution is the product of two 2-spheres $S^{(2)} \times S^{(2)}$, and in the limit when $r_{0} \gg l$, its action is given by Eq. (3.7) with $V_{4}^{(\gamma)}=(4 \pi / 3)^{2}$ and $r_{i}=r_{0}$ :

$$
S_{E}^{N} \approx-\frac{2 \pi}{3 G H^{2}} .
$$

Interestingly, this is the same as the action of the 4-dimensional Nariai instanton with the same curvature radius. For compact instantons, $-S_{E}$ coincides with the gravitational entropy. Hence, as long as the size of the instanton is large compared with the "cutoff" scale $l$, the entropy of the 5-dimensional solution coincides with the entropy of the brane world at the boundary. This is actually true for any choice of brane-world geometry, and is perhaps the consequence of a deeper holographic principle.

As mentioned above, the 5-dimensional instanton is singular at $r=0$. However, by introducing a boundary at some small $r$, the Hawking-Gibbons boundary term

$$
-\frac{a^{4}}{8 \pi G_{5}} \int \sqrt{\gamma} K_{\mu}^{\mu} d^{4} x=\frac{3 a^{3} \partial_{r} a}{16 \pi G_{5}} V_{4}^{(\gamma)}
$$

vanishes as $r \rightarrow 0$ (here $K$ is the extrinsic curvature). Hence, this boundary does not contribute to the action. Since the 
singularity is so mild, we believe that the Nariai-brane instanton indeed represents a legitimate saddle point giving the main contribution to the pair-creation rate of "black cigars" attached to the brane world. This rate is obtained by exponentiating the difference between Eq. (3.11) and the action for the de Sitter-brane solution:

$$
S_{E}^{d S} \approx-\frac{\pi}{G H^{2}}
$$

This gives the usual 4-dimensional result [20] $\Gamma \sim \exp$ $\left(-\pi / 3 G H^{2}\right)$.

In an alternative scenario, the singularity can be avoided by introducing a membrane of negative tension, as mentioned at the beginning of this section. For sufficiently large absolute value of the tension, the contribution of this second brane-world to the action will be negligible and we recover Eq. (3.11).

Finally, we may speculate about the existence of additional instantons describing the pair creation of black holes during inflation. One reason to suspect the existence of such instantons is that the black cigar on a flat brane has some corrections with respect to the Schwarzschild solution, whereas the instanton presented here has no such corrections with respect to the Nariai solution. However, it should be recalled that the mass of the black holes considered here is not arbitrary: it is related to the curvature of de Sitter and some "miraculous" cancellation of the corrections may occur for this special value of the mass. Nevertheless, we should leave the door open to the possible existence of completely non-singular solutions. If these existed, they may have slightly lower action than the ones we have considered here. However, even in this case we expect that their action would be very similar in the low energy limit (since in this limit the action of our instanton agrees with the fourdimensional Nariai action).

\section{AdS BLACK HOLES AND THERMAL INSTANTONS}

In this section we shall consider instanton solutions where the black hole is not on the brane, but in the bulk. We assume a scalar field as the matter on the brane. Since the instanton is compact, we may interpret these configurations as describing the birth of a brane world which contains a bulk black hole. An alternative interpretation is that they give the probability for "pair' creation of bulk black holes inside of a pre-existing inflating brane world, analogous to the creation of black holes in the presence of a domain wall in four dimensions [21-24]. This interpretation parallels the one usually attributed to the 4D Nariai solution.

The most general form for the spherically symmetric Euclidean space in the bulk is the Schwarzschild-AdS solution, given by

$$
d s^{2}=A(R) d T_{E}^{2}+\frac{d R^{2}}{A(R)}+R^{2} d \Omega_{(3)}^{2}
$$

Here

$$
A(R)=1+\frac{R^{2}}{l^{2}}-\frac{\alpha^{2}}{R^{2}}
$$

where the parameter $\alpha$ is related to the mass of the five dimensional black hole. A spherial brane can be introduced, with trajectory given by $R=R\left(t_{E}\right), T=T\left(t_{E}\right)$. Two identical copies of the bulk bounded by this trajectory are glued at the brane. Choosing the parameter $t_{E}$ to be the cosmological proper "time"' on the brane, the equations of motion reduce to the Euclidean version of Eq. (2.11),

$$
-\left(\frac{\dot{R}}{R}\right)^{2}=-\frac{1}{R^{2}}+\frac{\alpha^{2}}{R^{4}}+\left(X_{E}+\frac{l^{2}}{4} X_{E}^{2}\right) .
$$

Here $X_{E}=(8 \pi G / 3) \rho_{E}$, with $\rho_{E}=V(\phi)-(1 / 2) \dot{\phi}^{2}$ and the overdot indicates a derivative with respect to $t_{E}$. This equation should be supplemented with the equation of motion for the scalar field:

$$
\ddot{\phi}+3 \frac{\dot{R}}{R} \dot{\phi}=V^{\prime}(\phi)
$$

Taking the derivative of Eq. (4.2) and using Eq. (4.3) we obtain

$$
-\ddot{R}=\frac{-\alpha^{2}}{R^{3}}+R\left(X_{E}+\frac{l^{2}}{4} X_{E}^{2}\right)+4 \pi G \dot{\phi}^{2} R\left(1+\frac{l^{2}}{2} X_{E}\right) .
$$

Let $\phi_{0}$ be an extremum of $V(\phi)$. Then, it is straightforward to show that there is a static solution with $\phi=\phi_{0}$ and

$$
R_{0}^{2}=2 \alpha^{2}=\left[2\left(X_{0}+l^{2} X_{0}^{2} / 4\right)\right]^{-1} .
$$

The effective tension of the brane is given by $\sigma=\sigma_{c}$ $+V\left(\phi_{0}\right)$. Note that both the radius of the brane and the mass parameter $\alpha$ are given in terms of the energy density on the brane at the stationary point.

The bulk metric (4.1) has a conical singularity in the $\left(R, T_{E}\right)$ plane at $R=0$, unless the the range of the "angular", variable $T_{E}$ is adjusted as

$$
0<T_{E} \leqslant \beta=4 \pi \lim _{R \rightarrow R_{+}} \frac{\left(R-R_{+}\right)}{A(R)} .
$$

Here

$$
R_{+}^{2}=\frac{1}{2}\left(l \sqrt{l^{2}+4 \alpha^{2}}-l^{2}\right)
$$

is the black hole horizon radius. The temperature on the brane will be given by the inverse of the periodicity of the proper time:

$$
T_{b}=\beta^{-1} A^{-1 / 2}\left(R_{0}\right)=\frac{1}{\sqrt{2} \pi R_{+}} .
$$

Hence the temperature felt by observers on the brane is proportional to the inverse of the black hole radius. 
The Euclidean action of the thermal instanton can be calculated along the same lines discussed in Sec. II, and it is given by

$$
S_{E}=-\frac{\pi^{2} R_{+}^{3}}{G l}
$$

Note that this expression also follows from the area law. From the five-dimensional point of view, the entropy of this system is due to the area of the AdS black hole, which is equal to $4 \pi^{2} R_{+}^{3}$ (two copies of the black hole must be considered). Since $G_{5}=G l$, we have

$$
S_{b h}=-S_{E}=\text { area } / 4 G_{5} .
$$

Incidentally, the same argument can be applied to the de Sitter and Nariai instantons considered in the previous sections. There, the action is equal to minus one-fourth of the total area of cosmological and black hole horizons, in natural units.

For $\alpha \gg l$, the black hole radius, $R_{+}^{2} \approx \alpha l$, is much larger than the AdS radius. In that case, the action of this instanton is much larger (smaller in absolute value) than the action of the Nariai-brane instanton with the same value of the effective brane tension (or 4-dimensional cosmological constant). Hence, the nucleation rate of bulk black holes would be very suppressed compared with the nucleation rate of black cigars on the brane.

On the other hand, if the thermal instanton is interpreted as describing creation of a hot universe from nothing, it is of some interest to consider the evolution of this universe after creation. This is described by the analytic continuation of Eq. (4.2):

$$
\left(\frac{\dot{R}}{R}\right)^{2}=\frac{-1}{R^{2}}+\left(\frac{8 \pi G}{3}\right) \rho+l^{2}\left(\frac{4 \pi G}{3} \rho\right)^{2}+\frac{\alpha^{2}}{R^{4}},
$$

where $\rho$ is the density of matter on the brane. This is very similar to the usual Friedmann equation for the evolution of a 4-dimensional universe in Einstein gravity. The only differences are the third and fourth terms on the right hand side of the equation. The third term is the correction at large densities which we had already encountered in Sec. II. The last term, which is due to the presence of the black hole in the bulk, behaves from the point of view of four dimensions as an additional contribution to the matter density which dilutes like radiation.

The possible presence of an additional term which dilutes like radiation was noted in Ref. [11] for the case of spatially flat cosmology. There, the analogue of $\alpha$ appeared as an integration constant of the equations of motion on the brane. As shown above, this term corresponds to the existence of a black hole in the bulk $[22,25,26]$. In fact, any spherically symmetric massive object in the bulk (or even a "massive", parallel brane world concentrical with ours) would cause the same effect. This additional term does not arise in the case of the maximally symmetric de Sitter-brane instanton. In principle it would be present if the universe was born via the thermal instanton. However, since the universe must inflate after creation, it seems that in the post-inflationary epoch the most reasonable choice for the "integration constant" is zero.

Recently, there has been much interest in the so-called AdS/conformal field theory (CFT) correspondence, a possible duality relation between classical gravity in a five dimensional AdS bulk and a conformal field theory residing on its boundary [27]. In this context, the last term in Eq. (4.8) may be interpreted from the boundary theory point of view as the contribution of a true radiation bath of the conformal fields whose energy density is $\rho_{r}=(3 / 8 \pi G)\left(\alpha^{2} / R_{0}^{4}\right)$, whose temperature is $T_{b}$ and whose volume is given by $2 \pi^{2} R_{0}^{3}$. The entropy of such radiation bath is given by

$$
S_{\text {rad }}=\frac{\pi^{2} \alpha R_{+}}{G} .
$$

We note that for $\alpha \gg l$, this coincides with the entropy of the 5D bulk black hole given by Eq. (4.7),

$$
S_{\text {rad }}(4 D) \approx S_{b h}(5 D),
$$

in agreement with the duality conjecture.

\section{CONCLUSIONS}

We have presented a quantum cosmological scenario of brane-world creation. In this scenario, not only an inflating brane but also the associated 5-dimensional bulk spacetime is created from nothing. The quantum creation is described by the de Sitter-brane instanton which consists of two identical patches of anti-de Sitter space glued together by an $S^{(4)}$ brane. The Euclidean action of the instanton is generally different from that of the 4-dimensional de Sitter instanton but reduces to it if the de Sitter radius is large compared with the AdS curvature radius. We have found that the gravitational Kaluza-Klein modes have a mass gap in the spectrum and the continuous spectrum starts above the mass $m=(3 / 2) H$, where $H$ is the expansion rate during inflation.

We have also found that there is a family of 5-dimensional solutions such that the metric on the brane is any solution of the 4-dimensional vacuum Einstein equations with a cosmological constant. Unless the 4D metric is conformally flat, these solutions are singular at the center of the AdS bulk. But the singularity is so weak that it does not affect the value of the action at all (the singularity can also be avoided by placing a negative tension brane at a small radius without altering the Euclidean action much.) Among these solutions is the Nariai-brane instanton which has the topology of $S^{(2)} \times S^{(2)}$ on the brane. We have argued that this instanton describes the pair creation of black cigars threading through the brane.

We have studied thermal instantons which contain an AdS black hole in the bulk. These may either represent the creation of a hot universe from nothing or the pair creation of AdS black holes near an inflating brane. In the latter interpretation, however, the nucleation rate of AdS black holes would be very much suppressed compared with that of black cigars attached to the brane when the size of the instantons is larger than the AdS radius. The tidal forces exerted by such 
black holes on the brane manifest themselves as an additional term in the Friedmann equation which dilutes like radiation. In the spirit of the AdS/CFT correspondence, this may be attributed to thermal radiation in the boundary theory. In this case, for temperatures much lower than the AdS scale, the entropy of this radiation agrees with the entropy of the black hole in the five dimensional theory.

The fact that the Euclidean action of brane instantons reduces to the corresponding 4-dimensional action in the low energy limit is reassuring. In particular, this suggests that most of the predictions made in the standard 4-dimensional quantum cosmological context are qualitatively valid also in brane-world scenarios. An exception may occur for instantons describing the creation of open universes from nothing [28]. These instantons are singular in four dimensions, and therefore a different behavior of gravity at high densities and short distances can make a difference in the Euclidean action. This case is left for further study.

\section{ACKNOWLEDGMENTS}

We thank Tetsuya Shiromizu for useful discussions. J.G. acknowledges support from CICYT under grant AEN981093. M.S. acknowledges support from Monbusho, under Grant-in-Aid for Scientific Research No. 09640355. J.G. thanks X. Montes and T. Tanaka for useful discussions and the organizers of the 4th RESCEU symposium on "The Birth and Evolution of the Universe" for their kind hospitality during the initial stages of this work.
[1] See, e.g., J. Polchinski, String Theory I \& II (Cambridge University Press, Cambridge, England, 1998).

[2] N. Arkani-Hamed, S. Dimopoulos and G. Dvali, Phys. Lett. B 429, 263 (1998); Phys. Rev. D 59, 086004 (1999); I. Antoniadis, N. Arkani-Hamed, S. Dimopoulos and G. Dvali, Phys. Lett. B 436, 257 (1998); A. Pomarol, hep-ph/9911294.

[3] L. Randall and R. Sundrum, Phys. Rev. Lett. 83, 3370 (1999).

[4] L. Randall and R. Sundrum, Phys. Rev. Lett. 83, 4690 (1999).

[5] T. Shiromizu, K. Maeda and M. Sasaki, Phys. Rev. D 62, 024012 (2000).

[6] J. Garriga and T. Tanaka, hep-th/9911055.

[7] C. Csaki, M. Graesser, L. Randall and J. Terning, Phys. Rev. D 62, 045015 (2000); W. Goldberger and M. Wise, hep-th/9911457.

[8] A. Chamblin, S.W. Hawking and H.S. Reall, Phys. Rev. D 61, 065007 (2000).

[9] R. Emparan, G.T. Horowitz and R.C. Myers, J. High Energy Phys. 01, 007 (2000).

[10] N. Kaloper, Phys. Rev. D 60, 123506 (1999).

[11] P. Binétruy, C. Deffayet, U. Ellwanger and D. Langlois, hep-th/9910219; E.E. Flanagan, S.-H.H. Tye and I. Wasserman, hep-ph/9910498.

[12] We were informed by T. Shiromizu that the Lorentzian version of a family of spherically symmetric brane solutions has been recently discussed by Kraus [25] (see also [26]).

[13] H. Kodama and M. Sasaki, Suppl. Prog. Theor. Phys. 78, 1 (1984).

[14] J.B. Hartle and S.W. Hawking, Phys. Rev. D 28, 2960 (1983).

[15] A. Vilenkin, Phys. Rev. D 30, 509 (1984); 33, 3560 (1986).

[16] J. Garriga and A. Vilenkin, Phys. Rev. D 56, 2464 (1997).

[17] A. Chamblin and G.W. Gibbons, hep-th/9909130.
[18] M. Sasaki, T. Shiromizu and K. Maeda, Phys. Rev. D 62, 024008 (2000).

[19] R. Gregory and R. Laflamme, Phys. Rev. Lett. 70, 2837 (1993).

[20] P. Ginsparg and M.J. Perry, Nucl. Phys. B222, 245 (1983).

[21] R. Caldwell, A. Chamblin and G. Gibbons, Phys. Rev. D 53, 7103 (1996); R.B. Mann, Nucl. Phys. B516, 357 (1998); A. Chamblin and J.A.M. Ashbourn-Chamblin, Phys. Rev. D 57, 3529 (1998).

[22] The use of compact Euclidean solutions in order to describe nucleation processes in a preexisting background is the subject of some debate (see e.g. [23,24] for a recent discussion). The problem is that the instanton itself does not represent a semiclassical path interpolating between the original configuration and the configuration after tunneling (incidentally, this is true even for the well-known Coleman-de Lucchia instanton describing false vacuum decay in the presence of gravity). Here, we shall not delve into this problem, adopting the interpretation given in the original references. Our emphasis will be in the case corresponding to creation from nothing, where the interpretation of instantons as semiclassical paths is perhaps less controversial.

[23] R. Bousso and A. Chamblin, Phys. Rev. D 59, 084004 (1999).

[24] U. Gen and M. Sasaki, Phys. Rev. D 61, 103508 (2000).

[25] P. Kraus, J. High Energy Phys. 12, 011 (1999).

[26] M. Cvetic, S. Griffies and H.H. Soleng, Phys. Rev. D 48, 2613 (1993); M. Cvetic and H.H. Soleng, Phys. Rep. 282, 159 (1997); A. Chamblin and H. Reall, Nucl. Phys. B562, 133 (1999); D. Ida, gr-qc/9912002.

[27] See e.g. S.S. Gubser, hep-th/9912001, and references therein.

[28] S.W. Hawking and N. Turok, Phys. Lett. B 425, 25 (1998); 432, 271 (1998). 